\title{
TIME-DISCRETISED GALERKIN APPROXIMATIONS OF PARABOLIC STOCHASTIC PDES
}

\author{
W. Grecksch and P.E. Kloeden
}

The global discretisation error is estimated for strong time discretisations of finite dimensional Ito stochastic differential equations (SDEs) which are Galerkin approximations of a class of parabolic stochastic partial differential equation (SPDE) with a strongly monotone linear operator with eigenvalues $\lambda_{1} \leqslant \lambda_{2} \leqslant \cdots$ in its drift term. If an order $\gamma$ strong Taylor scheme with time-step $\Delta$ is applied to the $N$ dimensional Ito-Galerkin SDE, the discretisation error is bounded above by

$$
K\left(\lambda_{N+1}^{-1 / 2}+\lambda_{N}^{[\gamma+1 / 2]+1} \Delta^{\gamma}\right)
$$

where $[x]$ is the integer part of the real number $x$ and the constant $K$ depends on the initial value, bounds on the other coefficients in the SPDE and the length of the time interval under consideration.

\section{INTRODUCTION}

Numerical methods for parabolic stochastic partial differential equations (SPDEs) require the discretisation of both time and space variables. While both can be discretised simultaneously, the advantage of first discretising the state variables by either Galerkin or finite difference methods is that higher order numerical schemes that have been derived for finite dimensional Ito stochastic differential equations (SDEs) can then be applied in the time discretisation stage (see [3] and the references cited therein). The constants in the time discretisation error estimate then depend on the dimension of the SDE under consideration and the nature of this dependence needs to be clarified in order to provide a useful global space-time discretisation error estimate. This will be done here for a class of parabolic stochastic partial differential equations that includes special stochastic reaction-diffusion equations.

Let $\mathcal{D}$ be a bounded domain in $\mathbb{R}^{d}$ with sufficiently smooth boundary $\partial \mathcal{D}$ and consider a parabolic SPDE

$$
d U_{t}=\left\{A U_{t}+f\left(U_{t}\right)\right\} d t+g\left(U_{t}\right) d W_{t}
$$

Received 13th September, 1995.

Partially supported by the Australian Research Council Grant A 89601825 and a travel grant from the Deutsche Forschungsgemeinschaft.

Copyright Clearance Centre, Inc. Serial-fee code: 0004-9729/96 \$A2.00+0.00. 
where $\left\{W_{t}, t \geqslant 0\right\}$ is a standard scalar Wiener process, with Dirichlet boundary condition

$$
\left.U\right|_{\partial \mathcal{D}}=0
$$

and initial condition $U_{0} \in H_{0}^{1,2}(\mathcal{D})$. Here $H_{0}^{1,2}(\mathcal{D})$ is the space of functions $u: \mathcal{D} \mapsto \mathbb{R}^{1}$ which vanish on $\partial \mathcal{D}$ such that $u$ and its first order generalised derivatives $D u$ belong to $L_{2}(\mathcal{D})$ with the norm $\|\cdot\|$ and $A$ is a linear operator which is densely defined in $L_{2}(\mathcal{D})$ by $\left\{v \in H_{0}^{1,2}(\mathcal{D}): A v \in L_{2}(\mathcal{D})\right\}$ such that $-A$ is strongly monotone, that is there is a positive constant $\alpha$ such that

$$
(-A u, u) \geqslant \alpha\|u\|^{2}, \quad u \in H_{0}^{1,2}(\mathcal{D})
$$

where $(\cdot, \cdot)$ is the inner product and $\mid \cdot$ the associated norm of the space $L_{2}(\mathcal{D})$. In addition $f$ and $g$, which map either $L_{2}(\mathcal{D})$ or $H_{0}^{1,2}(\mathcal{D})$ into itself, are formed from real valued functions of a real variable with uniformly bounded derivatives of apropriate order, into which the numerical values $U=U(t, x, \omega)$ are inserted (thus derivatives of $U$ are not present here).

The eigenvalues $\lambda_{j}$ and corresponding eigenfunctions $\phi_{j} \in H_{0}^{1,2}(\mathcal{D})$ of the operator $-A$, that is with

$$
-A \phi_{j}=\lambda_{j} \phi_{j}, \quad j=1,2, \ldots
$$

form an orthonormal basis in $L_{2}(\mathcal{D})$ with $\lambda_{j} \rightarrow \infty$ as $j \rightarrow \infty$ (see [1]); for example, if $A$ is the Laplacian operator on the unit interval [0,1], then $\lambda_{j}=j^{2} \pi^{2}$ and $\phi_{j}=$ $\sqrt{2} \sin (j \pi x)$. Each $u \in L_{2}(\mathcal{D})$ thus has the unique representation $u=\sum_{j=1}^{\infty} u_{j} \phi_{j}$ with norm $|u|=\sqrt{\sum_{j=1}^{\infty} u_{j}^{2}}$, while each $u \in H_{0}^{1,2}(\mathcal{D})$ has the unique representation $u=$ $\sum_{j=1}^{\infty} \sqrt{\lambda_{j}} u_{j} \phi_{j}$ with equivalent norm $\|u\|=\sqrt{\sum_{j=1}^{\infty} \lambda_{j} u_{j}^{2}}$.

Let $\mathcal{X}_{N}$ be the $N$-dimensional subspace of $H_{0}^{1,2}(\mathcal{D})$ spanned by $\left\{\phi_{1}, \cdots, \phi_{N}\right\}$ and let $P_{N}$ denote the projection of $L_{2}$ or $H_{0}^{1,2}(\mathcal{D})$ onto $\mathcal{X}_{N}$. Write $U^{N}$ synonomously for $\left(U^{N, 1}, \cdots, U^{N, N}\right) \in \mathbb{R}^{N}$ and $\sum_{j=1}^{N} U^{N, j} \phi_{j} \in \mathcal{X}_{N}$ according to context and define $A_{N}=\left.P_{N} A\right|_{\mathcal{X}_{N}}, f_{N}=\left.P_{N} f\right|_{\mathcal{X}_{N}}$ and $g_{N}=\left.P_{N} g\right|_{\mathcal{X}_{N}}$ where $f$ and $g$ are now interpreted as mappings of $L_{2}(\mathcal{D})$ or $H_{0}^{1,2}(\mathcal{D})$ into itself. The $N$-dimensional ItoGalerkin SDE corresponding to the SPDE (1) and boundary condition (2) is then

$$
d U_{t}^{N}=\left\{A_{N} U_{t}^{N}+f_{N}\left(U_{t}^{N}\right)\right\} d t+g_{N}\left(U_{t}^{N}\right) d W_{t}
$$


Note that the effect of $A_{N} U^{N}$ is equivalent to multiplication of $U^{N} \in \mathbb{R}^{N}$ by an $N \times N$ diagonal matrix with $j t h$ diagonal component $-\lambda_{j}$.

Following Kloeden and Platen [3], an order $\gamma$ strong Taylor scheme with constant time-step $\Delta$ for the SDE (4) has the form

$$
Y_{k+1}^{N}=Y_{k}^{N}+\sum_{\alpha \in \mathcal{A} \gamma \backslash\{v\}} F_{\alpha}^{N}\left(Y_{k}^{N}\right) I_{\alpha, k, \Delta}
$$

with coefficient functions $F_{\alpha}^{N}$ and multiple stochastic integrals $I_{\alpha, k, \Delta}$, where $\gamma$ takes possible values 0.5 (Euler scheme), 1.0 (Milstein scheme), 1.5, 2.0, .. . The admissible multi-indices $\alpha=\left(j_{1}, \cdots, j_{l}\right) \in \mathcal{A}_{\boldsymbol{\gamma}} \backslash\{\boldsymbol{v}\}$ here have components $j_{i}=0$ or 1 corresponding to integration with respect to ' $d t$ ' or ' $d W_{t}$ ', respectively, while the $j t h$ component of $F_{\alpha}^{N}$ is defined by

$$
F_{\alpha}^{N, j}\left(Y^{N}\right)=L_{N}^{j_{1}} \cdots L_{N}^{j_{l}} F_{j}\left(Y^{N}\right)
$$

where the operators

$$
L_{N}^{0}=\sum_{j=1}^{N}\left(-\lambda_{j} U^{N, j}+f^{N, j}\left(U^{N}\right)\right) \frac{\partial}{\partial U^{N, j}}+\frac{1}{2} \sum_{i, j=1}^{N} g^{N, i}\left(U^{N}\right) g^{N, j}\left(U^{N}\right) \frac{\partial^{2}}{\partial U^{N, i} \partial U^{N, j}}
$$

and

$$
L_{N}^{1}=\sum_{j=1}^{N} g^{N, j}\left(U^{N}\right) \frac{\partial}{\partial U^{N, j}}
$$

are applied successively to $F_{j}\left(Y^{N}\right) \equiv Y^{N, j}$ and the result evaluated at $Y^{N}$. See [3] for further details.

For a common initial value $U_{0}^{N}=Y_{0}^{N}$ the global strong discretisation error of the numerical scheme (5) has the mean-square form

$$
E\left|U_{k \Delta}^{N}-Y_{k \Delta}^{N}\right|^{2} \leqslant K_{N} \Delta^{2 \gamma}
$$

where the constant $K_{N}$ depends on the dimension $N$ as well as on the time interval $0 \leqslant k \Delta \leqslant T$ under consideration and uniform bounds on the functions $f$ and $g$ and their derivatives. The main result of this paper will be to determine the nature of the dependence of the constant $K_{N}$ in (7) on $N$ and to combine it with the truncation error in the Galerkin approximation to obtain an estimate of the combined space-time discretisation error for the SPDE (1) for the numerical scheme (5) applied to the ItoGalerkin SDE (4). 


\section{Main Result}

Let $(\Omega, \mathcal{F}, P)$ be a probability space, let $\left\{\mathcal{F}_{t}, t \geqslant 0\right\}$ be a filtration contained in $\mathcal{F}$ with respect to the given Wiener process $\left\{W_{t}, t \geqslant 0\right\}$, and let $\mathcal{L}_{2}=L_{2}\left(\Omega, L_{2}(\mathcal{D})\right)$ and $\mathcal{H}_{0}^{1,2}=L_{2}\left(\Omega, H_{0}^{1,2}(\mathcal{D})\right)$ be spaces of mean-square integrable $L_{2}(\mathcal{D})$ and $H_{0}^{1,2}(\mathcal{D})$ valued $\mathcal{F}$-measurable random variables, that is with finite values of $E|u|^{2}$ and $E\|u\|^{2}$, respectively.

It is well known (see $[\mathbf{1}, \mathbf{2}]$ ) that the above SPDE (1) with boundary condition (2) then has a unique $\mathcal{F}_{t}$-measurable strong solution $U_{t}$ with

$$
U \in L_{2}\left([0, T], \mathcal{H}_{0}^{1,2}\right) \bigcap C\left([0, T], \mathcal{L}_{2}\right)
$$

for each finite $T>0$ and initial condition $U_{0} \in \mathcal{H}_{0}^{1,2}$, since by the above assumptions $f$ and $g$ are uniformly Lispchitz continuous from $L_{2}(\mathcal{D})$ into itself and thus satisfy a linear growth bound on $L_{2}(\mathcal{D})$. By similar arguments, a unique solution $U_{t}^{N}$ also exists for the Ito-Galerkin $\operatorname{SDE}(4)$ with the initial value $U_{0}^{N}=P_{N} U_{0}$. For the remainder of the paper it will be assumed that the iterates $Y_{k}^{N}$ of the numerical scheme (5) have initial value $Y_{0}^{N}=P_{N} U_{0}$ and belong to $\mathcal{X}_{N}$ rather than $\mathbb{R}^{N}$.

THEOREM 1. The global space-time discretisation error of the order $\gamma$ strong Taylor scheme (5) with constant time-step $\Delta$ applied to the $N$-dimensional ItoGalerkin approximation (4) of the SPDE (1) has the form

$$
E\left|U_{k \Delta}-Y_{k}^{N}\right| \leqslant K\left(\lambda_{N+1}^{-1 / 2}+\lambda_{N}^{[\gamma+1 / 2]+1} \Delta^{\gamma}\right)
$$

where $[x]$ is the integer part of the real number $x$ and the constant $K$ depends on $E\left\|U_{0}\right\|^{2}$, bounds on the $f, g$ coefficients of the SPDE and the length of the time interval $0 \leqslant k \Delta \leqslant T$ under consideration.

The proof will be presented in the next section. It can be strengthened as in [3] by use of Doob inequalities to provide a similar estimate for $E \max _{0 \leqslant k \leqslant T / \Delta}\left|U_{k \Delta}-Y_{k}^{N}\right|$, but this will not be done here.

\section{ProOF}

The inequalities

$$
\left|P_{N} u\right| \leqslant|u|, \quad\left|\left(I-P_{N}\right) u\right| \leqslant \lambda_{N+1}^{-1 / 2}\|u\|
$$

hold since

$$
\left|P_{N} u\right|^{2}=\sum_{j=1}^{N} u_{j}^{2} \leqslant \sum_{j=1}^{\infty} u_{j}^{2}=|u|^{2}
$$


and

$$
\left|\left(I-P_{N}\right) u\right|^{2}=\sum_{j=N+1}^{\infty} u_{j}^{2} \leqslant \lambda_{N+1}^{-1} \sum_{j=N+1}^{\infty} \lambda_{j} u_{j}^{2} \leqslant \lambda_{N+1}^{-1} \sum_{j=1}^{\infty} \lambda_{j} u_{j}^{2} \leqslant \lambda_{N+1}^{-1}\|u\|^{2} .
$$

Also if $U \in H_{0}^{1,2}(\mathcal{D})$ and $U^{N} \in \mathcal{X}_{N}$, then

$$
\begin{aligned}
\left(A U-A_{N} U^{N}, U-U^{N}\right) & =\left(A\left(U-U^{N}\right), U-U^{N}\right) \\
& \leqslant-\alpha\left\|U-U^{N}\right\|^{2}
\end{aligned}
$$

since $A_{N}=A$ on $\mathcal{X}_{N}$ and $-A$ is strongly monotone and linear. Hence by the Ito formula it follows for the solutions $U_{t}$ of (1) and $U_{t}^{N}$ of (4) that

$$
\begin{aligned}
E\left|U_{t}-U_{t}^{N}\right|^{2}=E & \left|U_{0}-U_{0}^{N}\right|^{2}+2 E \int_{0}^{t}\left(A U_{s}-A_{N} U_{s}^{N}, U_{s}-U_{s}^{N}\right) d s \\
& +2 E \int_{0}^{t}\left(f\left(U_{s}\right)-f_{N}\left(U_{s}^{N}\right), U_{s}-U_{s}^{N}\right) d s \\
& +2 E \int_{0}^{t}\left|g\left(U_{s}\right)-g_{N}\left(U_{s}^{N}\right)\right|^{2} d s \\
\leqslant & E\left|\left(I-P_{N}\right) U_{0}\right|^{2}-2 \alpha E \int_{0}^{t}\left\|U_{s}-U_{s}^{N}\right\|^{2} d s \\
& +2 E \int_{0}^{t}\left|f\left(U_{s}\right)-f_{N}\left(U_{s}^{N}\right)\right|\left|U_{s}-U_{s}^{N}\right| d s \\
& +2 E \int_{0}^{t}\left|g\left(U_{s}\right)-g_{N}\left(U_{s}^{N}\right)\right|^{2} d s .
\end{aligned}
$$

Now by the inequalities (9) and the fact that $f$ is uniformly Lipschitz with constant $K$ as a mapping from $L_{2}(\mathcal{D})$ into itself

$$
\begin{aligned}
\left|f(U)-f_{N}\left(U^{N}\right)\right|^{2} & =\left|f(U)-P_{N} f\left(U^{N}\right)+P_{N} f(U)-f_{N}\left(U^{N}\right)\right|^{2} \\
& \leqslant 2\left|f(U)-P_{N} f(U)\right|^{2}+2\left|P_{N} f(U)-f_{N}\left(U^{N}\right)\right|^{2} \\
& =2\left|\left(I-P_{N}\right) f(U)\right|^{2}+2\left|P_{N}\left(f(U)-f\left(U^{N}\right)\right)\right|^{2} \\
& \leqslant 2 \lambda_{N+1}^{-1}|f(U)|^{2}+2 K^{2}\left|U-U^{N}\right|^{2}
\end{aligned}
$$

with a similar inequality holding for $g$ too. Inserting these into the energy inequality 
above then gives

$$
\begin{aligned}
E\left|U_{t}-U_{t}^{N}\right|^{2} \leqslant & \lambda_{N+1}^{-1} E\left\|U_{0}\right\|^{2}+2 \lambda_{N+1}^{-1} E \int_{0}^{t}\left\|f\left(U_{s}\right)\right\| d s+4 \lambda_{N+1}^{-1} E \int_{0}^{t}\left\|g\left(U_{s}\right)\right\|^{2} d s \\
& +\left(1+6 K^{2}\right) E \int_{0}^{t}\left|U_{s}-U_{s}^{N}\right|^{2} d s \\
\leqslant & \lambda_{N+1}^{-1}\left(E\left\|U_{0}\right\|^{2}+2 E \int_{0}^{t}\left\|f\left(U_{s}\right)\right\| d s+4 E \int_{0}^{t}\left\|g\left(U_{s}\right)\right\|^{2} d s\right) \\
& +\left(1+6 K^{2}\right) E \int_{0}^{t}\left|U_{s}-U_{s}^{N}\right|^{2} d s
\end{aligned}
$$

so by the Gronwall inequality

$$
\begin{aligned}
& E\left|U_{t}-U_{t}^{N}\right|^{2} \\
& \quad \leqslant \lambda_{N+1}^{-1} e^{\left(1+6 K^{2}\right) T}\left(E\left\|U_{0}\right\|^{2}+E \int_{0}^{T}\left\|f\left(U_{s}\right)\right\| d s+4 E \int_{0}^{T}\left\|g\left(U_{s}\right)\right\|^{2} d s\right),
\end{aligned}
$$

that is,

$$
E\left|U_{t}-U_{t}^{N}\right|^{2} \leqslant K_{T, U_{0}} \lambda_{N+1}^{-1}
$$

where $K_{T, U_{0}}$ is independent of $N$.

Inequalities (7) and (10) combine to give the following global space-time discretisation error estimate

$$
E\left|U_{k \Delta}-Y_{k \Delta}^{N}\right|^{2} \leqslant 2 K_{T, U_{0}} \lambda_{N+1}^{-1}+2 K_{N} \Delta^{2 \gamma}
$$

To determine the nature of the dependence of $K_{N}$ on $N$ here it is necessary to examine the terms in the remainder of the strong stochastic Taylor expansion used to derive the strong Taylor scheme (5). Details are given in the proof of [3, Theorem 10.6.3] and will not be repeated in full here. In particular, the remainder consists of multiple stochastic integrals with nonconstant integrands $I_{\alpha, k, \Delta}\left(F_{\alpha}^{N}\left(U^{N}\right)\right)$ for multi-indices $\alpha$ in the remainder set $\mathcal{B}_{\gamma}$. These are estimated in inequality $[3$, p.364 (6.23)] under the assumption that the coefficient functions satisfy a linear growth bound. This is true here under the assumptions on the coefficients of the SPDE (1) and it is only the $\lambda_{j}(j=1$, $\ldots, N)$ factors in the operator $L_{N}^{0}$ that contribute to the dependence of the coefficient $K_{N}$ on $N$. Specifically, each application of $L_{N}^{0}$ contributes a single dominating power of $\lambda_{N}$. Let $l(\alpha)$ be the length of a multi-index $\alpha$ and $n(\alpha)$ the number of component indices $j_{i}$ that are equal to 0 . The bound in the squared inequality $[3$, p.364 (6.23)] thus takes the form

$$
K_{T}\left(1+E\left|U_{0}^{N}\right|^{2}\right) \lambda_{N}^{2 n(\alpha)} \Delta^{\phi(\alpha)}
$$


where $\phi(\alpha)$ is equal to $2(l(\alpha)-1)$ if $l(\alpha)=n(\alpha)$ and $l(\alpha)+n(\alpha)-1$ if $l(\alpha) \neq n(\alpha)$. If $\gamma$ is an integer the dominant value occurs for $l(\alpha)=n(\alpha)=\gamma+1$ and the required bound is of the form

$$
K_{T, U_{0}} \lambda_{N}^{2 \gamma+2} \Delta^{2 \gamma}
$$

while if $2 \gamma$ but not $\gamma$ is an integer the dominant value occurs for $l(\alpha)=n(\alpha)=\gamma+3 / 2$ and the required bound is of the form

$$
K_{T, U_{0}} \lambda_{N}^{2 \gamma+3} \Delta^{2 \gamma}
$$

Inserting this into (11), using the inequality $\sqrt{a^{2}+b^{2}} \leqslant|a|+|b|$ and renaming the constants, gives the desired result

$$
E\left|U_{k \Delta}-Y_{k \Delta}^{N}\right| \leqslant K_{T, U_{0}}\left(\lambda_{N+1}^{-1 / 2}+\lambda_{N}^{[\gamma+1 / 2]+1} \Delta^{\gamma}\right)
$$

where $[x]$ is the integer part of the real number $x$.

\section{REFERENCES}

[1] G. Da Prato and G. Zabczyk, Stochastic equations in infinite dimensions (Cambridge University Press, Cambridge, 1992).

[2] W. Grecksch and C. Tudor, Stochastic evolution equations (Akademie-Verlag, Berlin, 1995).

[3] P.E. Kloeden and E. Platen, Numerical solution of stochastic differential equations (Springer-Verlag, Berlin, Heidelberg, New York, 1992).

Institut für Optimierung und Stochastik Martin-Luther-Universität Halle-Wittenberg D-06099 Hale (Saale) Germany
School of Computing and Mathematics Deakin University, Geelong Campus Geelong Vic. 3217

Australia 